\title{
Measurement of effective atomic numbers using energy-resolved computed tomography
}

\section{$\operatorname{AUTHOR}(\mathrm{S}):$}

Yamashita, Yoshiki; Kimura, Masashi; Kitahara, Masaru; Hamaguchi, Takumi; Kanno, Ikuo; Ohtaka, Masahiko; Hashimoto, Makoto; Ara, Kuniaki; Onabe, Hideaki

\section{CITATION:}

Yamashita, Yoshiki ... [et al]. Measurement of effective ato mic numbers using energyresolved computed tomography. Journal of Nuclear Science and Technology 2014, 51(10): 1256-1263

\section{ISSUE DATE:}

2014-05-20

URL:

http://hdl.handle.net/2433/198799

\section{RIGHT:}

This is an Accepted Manuscript of an article published by Taylor \& Francis in "Journal of Nuclear Science and Technology" on 20 May 2014, available online: http://www.tandfonline.com/10.1080/00223131.2014.919881.; This is not the published version. Please cite only the published version.; この論文は出版社版でありません。引用の際には出版社 版をご確認ご利用ください。 


\title{
ARTICLE \\ Measurement of effective atomic numbers using energy-resolved computed tomography
}

\author{
Yoshiki YAMASHITA $^{\mathrm{a}^{*}}$, Masashi KIMURA ${ }^{\mathrm{a}}$, Masaru KITAHARA ${ }^{\mathrm{a}}$, Ikuo KANNO ${ }^{\mathrm{a}}$ \\ Masahiko OHTAKA ${ }^{\mathrm{b}}$, Makoto HASHIMOTO ${ }^{\mathrm{b}}$, Kuniaki ARA ${ }^{\mathrm{b}}$ and Hideaki ONABEc \\ ${ }^{a}$ Graduate School of Engineering, Kyoto University, Nishikyo, Kyoto 615-8530, Japan \\ ${ }^{\mathrm{b}} \mathrm{O}$-arai Research and Development Institute, Japan Atomic Energy Agency, Narita, O-arai, Ibaraki \\ 311-1393, Japan \\ ${ }^{\mathrm{c}}$ Raytech Corporation, Yoto, Utsunomiya 321-0904, Japan \\ (Received \\ )
}

For ion beam radiation therapy, the measurement of effective atomic numbers is necessary to know the material distribution in a human body: the range of ion beams entering the human body is influenced by the material distribution along their paths. Effective atomic numbers, however, cannot be measured at hospitals because monochromatic X-rays with different energies are necessary and are used only at synchrotron facilities. To make the effective atomic number measurements at hand, we propose energy-resolved computed tomography (CT) using a "transXend detector". By assigning two narrow energy ranges in the unfolding process of the data obtained by the transXend detector, the effective atomic numbers for acrylic and aluminum can be estimated by energy-resolved CT. The estimated effective atomic numbers are compared with those obtained by dual-energy and monochromatic 
X-ray CT.

Keywords: X-ray; computed tomography; effective atomic number; energy-resolved CT; white X-ray

*Corresponding author. E-mail: yamashita.yoshiki.35c@st.kyoto-u.ac.jp 


\section{Introduction}

For ion beam radiotherapy, the knowledge of the electron density distribution in a patient is important to determine the range of incident ions. The electron density distribution is estimated using the effective atomic number distribution, which can be obtained by linear attenuation coefficients measured using computed tomography (CT) using two monochromatic X-rays with different energies. For a monochromatic X-ray, the distribution of $\mu / \rho \times \rho^{*}$ in the subject is obtained for X-rays with a specific energy, where $\mu / \rho$ is the mass-attenuation coefficient and $\rho^{*}$ is the density of the material. Monochromatic X-rays, however, can be used at synchrotron radiation facilities, but not at hospitals. The distribution of the electron density cannot be employed for cancer treatment planning at this stage.

We report on an energy-resolved CT method using white X-rays, which were measured as electric current. The energy distribution of incident X-rays were obtained after analysis [1]. This method gives the X-ray energy distribution with arbitrarily chosen energy ranges. By narrowing the energy range, energy-resolved CT can therefore be performed using quasi-monochromatic X-rays.

This paper reports the resulting measurements of effective atomic numbers of acrylic and aluminum using energy-resolved CT. Acrylic and aluminum are the substitute materials for soft tissue and bone, respectively. These effective atomic numbers are compared with the ones measured by dual-energy and monochromatic X-ray CT.

\section{Estimation of effective atomic number}

The effective atomic number can be estimated using the linear attenuation coefficients of a material $\mu(E)$ at two different energies [2]. The X-ray linear attenuation coefficient of a material at an energy $E$ is described in terms of a photoelectric absorption cross-section $\tau(Z, E)$, a scattering cross-section $\sigma(Z, E)$ and an electron-pair production 
cross-section $\kappa(Z, E)$ as follows.

$$
\mu(E)=\rho \frac{N_{A}}{A}\{\tau(Z, E)+\sigma(Z, E)+\kappa(Z, E)\} .
$$

Here $\rho, N_{\mathrm{A}}$ and $A$ are the density, Avogadro's number and atomic mass of the material, respectively. Among the three reactions described above, electron-pair production does not occur in the energy range of $\mathrm{X}$-ray diagnosis. As a result, the ratio of the linear attenuation coefficient $\mu\left(E_{1}\right) / \mu\left(E_{2}\right)$ is described as

$$
\frac{\mu\left(E_{1}\right)}{\mu\left(E_{2}\right)}=\frac{\tau\left(E_{1}, Z\right)+\sigma\left(E_{1}, Z\right)}{\tau\left(E_{2}, Z\right)+\sigma\left(E_{2}, Z\right)} .
$$

In equation (2), the unknown value is the atomic number $Z$ if the linear attenuation coefficients are measured by X-rays with energies $E_{1}$ and $E_{2}$. Using the relationship between $\mu\left(E_{1}\right) / \mu\left(E_{2}\right)$ and $Z$, which is obtained by the literature, the effective atomic number $Z$ can be determined.

\section{Experiments}

\subsection{Experimental setup}

The experimental setup is shown in Figure 1. The X-ray tube (TRIX-150S, Toreck Co., Ltd., Japan) has a 2-mm-thick inherent Al filter to reduce low-energy X-rays. The tube voltage and current are $120 \mathrm{kVp}$ and $2.4 \mathrm{~mA}$, respectively. A Pb collimator with a diameter of $5 \mathrm{~mm}$ and thickness of $5 \mathrm{~mm}$ is attached to the exit aperture of the X-ray tube. The transXend detector is placed at the end of the X-ray path.

The transXend detector consists of four segment detectors made of $\mathrm{Si}(\mathrm{Li})$, which are aligned along the direction of X-ray incidence. The dimensions of the $\mathrm{Si}(\mathrm{Li})$ segment detector are $10 \times 10 \times 1 \mathrm{~mm}^{3}$. The $\mathrm{Si}(\mathrm{Li})$ segment detectors are operated without applying a bias voltage at room temperature. Furthermore, the transXend detector has a $58-\mu \mathrm{m}$-thick Sn filter in front of the third segment detector [3]. X-rays are measured as electric current by the 
segment detectors. After an unfolding process using previously obtained response functions, the X-ray energy distribution is estimated. In this study, six energy ranges are defined as shown in Table 1. The energy ranges $E_{2}$ and $E_{5}$ have the narrowest energy widths, $0.5 \mathrm{keV}$. We recognize $\mathrm{X}$-rays in these two energy ranges as quasi-monochromatic.

The response functions are obtained by measuring X-rays, which pass through aluminum and acrylic of known thicknesses. A step aluminum phantom is placed between the $\mathrm{X}$-ray tube and the transXend detector. The thicknesses of the step aluminum phantom change from 1-4 mm in 1-mm intervals in the direction of the X-ray path. In addition, the thickness of acrylic is changed from $0-30 \mathrm{~mm}$ using acrylic slabs with thicknesses of $10 \mathrm{~mm}$. By expressing the thickness of aluminum and acrylic as $k(k=0,4)$ and $l(l=0,3)$, respectively, the phantom is described as $m=(k, l)$.

The response functions $R_{i, j}$ are obtained by unfolding the following matrix equation using SANDII code [4]:

$$
\left(\begin{array}{c}
I_{i, 1} \\
I_{i, 2} \\
\cdot \\
I_{i, m}
\end{array}\right)=\left(\begin{array}{ccc}
Y_{1,1} & \cdot & Y_{6,1} \\
Y_{1,2} & \cdot & \cdot \\
\cdot & \cdot & \cdot \\
Y_{1, m} & \cdot & Y_{6, m}
\end{array}\right)\left(\begin{array}{c}
R_{i, 1} \\
R_{i, 2} \\
\cdot \\
R_{i, 6}
\end{array}\right)
$$

Here, $I_{i, m}$ is the electric current measured by the $i$-th $(i=1,4)$ segment detector when the phantom condition is $m . Y_{j, m}$ represents the calculated X-ray events in the energy range $E_{j}$ after passing the phantom $m$, and the X-ray energy spectrum is calculated by the formula of Birch et al. [5]. $R_{i, j}$ is the response function of the segment detector $i$ in the energy range $j$. By interpolating the measured current as a function of acrylic thickness, the response functions are estimated in 1-mm-thick intervals. 


\subsection{CT measurements}

The experimental setup for CT measurements is the same as the one shown in Figure 1, but the aluminum phantom and acrylic slabs are replaced by a cylindrical acrylic phantom with a diameter of $20 \mathrm{~mm}$. The cylindrical phantom has a 2-mm diameter aluminum rod in the center as shown in Figure 2. CT measurements are performed by the first-generation CT, i.e., by translating and rotating the phantom. The translation $(x)$ was carried out with steps of 0.2 mm and was repeated 115 times. The translation data was duplicated 17 times to have rotation data for every $10^{\circ}$ as the phantom had axial symmetry. By duplicating translation data, the fluctuation of X-ray intensity as a function of elapsed time was avoided.

Before starting the unfolding process, the X-ray path length was estimated for each measurement point $(x, \theta)$ using the CT image obtained with the current-measurement CT data. The X-ray path length was necessary to choose response functions that were prepared as a function of the acrylic thickness through which the X-rays passed. With measured electric current data, the X-ray energy distribution for each measurement point was calculated by unfolding the following matrix using the SANDII code:

$$
\left(\begin{array}{c}
I_{1, n} \\
I_{2, n} \\
\cdot \\
I_{4, n}
\end{array}\right)=\left(\begin{array}{cccc}
R_{1,1} & R_{1,2} & \cdot & R_{1,6} \\
R_{2,1} & \cdot & & \cdot \\
\cdot & & \cdot & \cdot \\
R_{4,1} & \cdot & \cdot & R_{4,6}
\end{array}\right)\left(\begin{array}{c}
Y_{1, n} \\
Y_{2, n} \\
\cdot \\
Y_{6, n}
\end{array}\right)
$$

Here, $n$ is the measurement point for a given $(x, \theta) . I_{i, n}$ is the electric current value measured by the $i$-th segment detector at measurement point $n . Y_{j, n}$ denotes the $\mathrm{X}$-ray events in the energy range $E_{j}$ after passing the phantom at measurement point $n$. CT images are reconstructed by $\mathrm{X}$-ray events in the energy ranges $E_{2}$ and $E_{5}$ with the filtered back projection method [6]. 


\subsection{Dual-energy CT}

The dual-energy CT measurements are carried out with X-ray tube voltages of 60 and $120 \mathrm{kVp}$. The tube currents are $2.0 \mathrm{~mA}$. The phantom and the experimental setup of the dual-energy CT measurements are the same as described in the previous section. The CT images are reconstructed by the electric current values measured by the first segment detector of the transXend detector.

\section{Results and Discussion}

\subsection{Effective atomic numbers}

Figure 3 shows the energy-resolved CT images reconstructed using (a) $Y_{2}$ and (b) $Y_{5}$. The CT-values in Figure 3 are the values that are proportional to the linear attenuation coefficients; they are not expressed in Hounsfield units. By dividing the CT-values of Figure 3 (a) by the corresponding values of Figure 3 (b), the linear attenuation coefficient ratios $\mu\left(E_{2}\right) / \mu\left(E_{5}\right)$ are obtained. The profile of a linear attenuation coefficient ratio along the diameter is shown in Figure 4. Based on the table of the mass-attenuation coefficients of elements given by the National Institute of Standard and Technology [7], the graph of the relationship between the linear attenuation coefficient ratio $\mu\left(E_{2}\right) / \mu\left(E_{5}\right)$ and atomic numbers is drawn in Figure 5. The ratios of linear attenuation coefficients of acrylic and aluminum are obtained by averaging them along the radii of 0.5 and $5.0 \mathrm{~mm}$, which were $1.14 \pm 0.03$ and $1.56 \pm 0.01$, respectively. Here, the errors 0.03 and 0.01 show the standard deviations of the ratios along the radii. With the experimental results obtained by energy-resolved CT, the effective atomic numbers of acrylic and aluminum are estimated as $6.70 \pm 0.54$ and $12.9 \pm 0.11$, respectively. The effective atomic number of acrylic is 6.47 in the literature [8].

The effective atomic numbers of acrylic and aluminum are obtained by dual-energy CT in the same way. The current CT images obtained by X-rays with tube voltages of (a) 60 
$\mathrm{kVp}$ and (b) $120 \mathrm{kVp}$ are shown in Figure 6. The averaged energies of X-rays for each tube voltage are 35.0 and $52.5 \mathrm{keV}$, respectively. The profile of the ratio of the linear attenuation coefficients $\mu(35.0) / \mu(52.5)$ along the diameter is shown in Figure 7. The averaged ratios of the linear attenuation coefficients of acrylic and aluminum are obtained in the same way as describe above, which were $1.29 \pm 0.21$ and $1.82 \pm 0.01$, respectively. The relationship between $\mu(35.0) / \mu(52.5)$ and atomic numbers is shown in Figure 8. The effective atomic numbers of acrylic and aluminum measured by dual-energy CT are estimated as $6.43 \pm 0.21$ and $9.96 \pm 0.03$.

\subsection{Effective atomic numbers as a function of energy range width}

The estimations of the effective atomic numbers described in the previous section were performed with the energy range width of $0.5 \mathrm{keV}$, i.e., 49.5-50.0 keV and 69.0-69.5 $\mathrm{keV}$. Keeping the upper limits of energy ranges constant, i.e., 50.0 and $69.5 \mathrm{keV}$, the estimation of the effective atomic numbers are carried out while changing the energy range widths: 1, 5, 10 and $15 \mathrm{keV}$. The CT images, the ratios of linear attenuation coefficients and the graphs of the relationships between linear attenuation coefficient and atomic numbers are made for each energy range width following the procedure described in Section 4.1. The estimation results are shown in Figure 9.

In the same way, the effective atomic numbers are obtained for the same energy widths described above by calculation. The atomic numbers obtained by calculations have nearly the same values despite the employed energy range widths. On the other hand, experimentally obtained atomic numbers showed a slight energy width dependence: by using wide energy widths, the estimated atomic numbers deviate from the correct ones. The reason for this energy width dependence is not understood. The fact that the estimated atomic numbers showed constant values with the employed energy width of $<5 \mathrm{keV}$, however, is encouraging in that it shows that monochromatic X-rays are not always necessary for the 
estimation of atomic numbers.

\subsection{Comparison of energy-resolved, dual-energy and monochromatic X-ray CTs}

The effective atomic number of water measured by monochromatic X-ray CT was reported by Torikoshi et al. [2]. They carried out measurements using monochromatic X-rays with energies of 40 and $70 \mathrm{keV}$. The rotation angle and translation steps were $0.8^{\circ}$ and $1 \mathrm{~mm}$, respectively. The phantom was an ellipsoidal vessel of $12 \times 16 \mathrm{~cm}^{2}$. The estimated and theoretical effective atomic numbers of water were 7.39 and 7.54, respectively.

Table 2 shows the estimated atomic numbers and relative errors obtained by energy-resolved, dual-energy and monochromatic X-ray CTs. The relative error is defined by $\left|1-Z_{\text {eff }} / Z\right|$. Here $Z_{\text {eff }}$ and $Z$ are the estimated and theoretical atomic numbers, respectively. For acrylic and water, the effective atomic numbers are estimated with the relative errors of a certain percentage by the three methods. On the other hand, the effective atomic number of aluminum measured by energy-resolved CT is far better than that by the dual-energy CT.

For atomic number estimation using dual-energy CT, the averaged energy $\bar{E}$ is obtained by

$$
\bar{E}=\int E \cdot Y(E) d E / \int Y(E) d E .
$$

The measured linear attenuation coefficient $\bar{\mu}$ is represented by

$$
\bar{\mu}=\int \mu(E) Y(E) d E / \int Y(E) d E .
$$

The spectrum weighted averages of the energy and the linear attenuation coefficient have those values at different energies. This is the reason why the relative errors are larger in the measurements using dual-energy CT: the small error for acrylic was obtained by chance.

\section{Conclusion}

Measurements of effective atomic numbers are typically performed by CT 
measurements with two different monochromatic energies or two tube voltages. The energy-resolved CT with the transXend detector enabled measurement of effective atomic numbers with white X-rays and only one exposure. The obtained results were comparable with those measured with monochromatic X-rays.

In the future, effective atomic number measurements using energy-resolved CT should be carried out for materials other than those employed for response function estimation and for compounds and mixtures.

\section{Acknowledgements}

The authors are grateful to Prof. K. Hitomi of Tohoku University for allowing them to use his CT image reconstruction program. Part of this study is supported by a Grant-in-Aid for Scientific Research from the Japan Society for the Promotion of Science, and the Suzuken Memorial Foundation. 


\section{References}

[1] Kanno I, Imamura R,Mikami K, Uesaka A, Hashimoto M, Ohtaka M, Ara K, Nomiya S, Onabe H. [A current mode detector for unfolding X-ray energy distribution]. J Nucl Sci Technol. 2008 Nov; 45:1165-1170.

[2] M. Torikoshi, T. Tsunoo, M. Sasaki, M. Endo, Y. Noda, Y. Ohno, T. Kohno, K. Hyodo, K. Uesugi, N. Yagi. [Electron density measurement with dual-energy X-ray CT using synchrotron radiation]. Phys. Med. Biol. 2003; 48:673-685.

[3] Kanno I, Shimazaki H, Imamura R, Yamashita Y, Shima K, Ohtaka M, Hashimoto M, Ara K, Nomiya S, Onabe H. [Low dose exposure diagnosis with a transXend detector aiming for iodine-marked cancer detection]. J Nucl Sci Technol. 2012;49:937-946.

[4] McElroy W, Berg S, Crockes T, Hawkins G. A computer-automated iterative method for neutron flux spectra determination by foil activation. New Mexico: Air Force Weapons Laboratory; 1967. p.8-16.

[5] Birch B, Marshall M. [Computation of Bremsstrahlung X-ray spectra and comparison with spectra measured with a Ge(Li) detector]. Phys Med Biol. 1917;24:505-517.

[6] Shepp LA, Logan B F. [Fourier reconstruction of a head section]. IEEE Trans Nucl Sci 1974; NS21-3: 21-43.

[7] Hubbell JH, Seltzer SM. Tables of X-ray mass attenuation coefficients and mass energy-absorption coefficients from $1 \mathrm{keV}$ to $20 \mathrm{MeV}$ for elements $\mathrm{Z}=1$ to 92 and 48 additional substances of dosimetric interest. The Physical Measurement Laboratory, The National Institute of Standard and Technology; 2010. Available from: http://www.nist.gov /pml/data /xraycoef / index.cfm.

[8] Mitchell MG, Emmanuel GC, Sandra CL. [Accuracies of the synthesized monochromatic $\mathrm{CT}$ numbers and effective atomic numbers obtained with a rapid $\mathrm{kVp}$ switching dual energy CT scanner]. Med Phys. 2011; 38:2222-2232. 


\section{Table captions}

Table 1 Assigned energy ranges (keV).

Table 2 Effective atomic numbers and their relative errors (RE) measured by energy-resolved, dual-energy and monochromatic X-ray CTs.

\section{Figure captions}

Figure 1. Experimental setup for response function estimation.

Figure 2. $\quad$ Schematic drawing of a cylindrical phantom.

Figure 3. $\quad$ CT images obtained by X-rays in the energy ranges (a) $E_{2}$ and (b) $E_{5}$.

Figure 4. Profile of the linear attenuation coefficients ratio along the diameter of the phantom.

Figure 5. Relationship between $\mu\left(E_{2}\right) / \mu\left(E_{5}\right)$ and atomic number.

Figure 6. CT images obtained by current-measurement CT value with the X-ray tube voltages of (a) $60 \mathrm{kVp}$ and (b) $120 \mathrm{kVp}$.

Figure 7. Profile of the linear attenuation coefficients ratio along the diameter of the phantom using the dual-energy CT. 
Figure 8. Relationship between the ratio of linear attenuation coefficients and atomic number using dual-energy CT.

Figure 9. The effective atomic numbers as a function of those obtained by experiments (solid) and simulation calculation (open) energy range width for aluminum (squares) and acrylic (diamonds). 
Table 1 Assigned energy ranges (keV).

\begin{tabular}{cccccc}
\hline$E_{1}$ & $E_{2}$ & $E_{3}$ & $E_{4}$ & $E_{5}$ & $E_{6}$ \\
\hline $15.0-49.5$ & $49.5-50.0$ & $50.0-60.0$ & $60.0-69.0$ & $69.0-69.5$ & $69.5-120.0$ \\
\hline
\end{tabular}


Table 2 Effective atomic numbers and their relative errors (RE) measured by energy-resolved, dual-energy and monochromatic X-ray CTs.

\begin{tabular}{c|c|c|c|c|c|c}
\hline & \multicolumn{2}{|c|}{ Energy-resolved } & \multicolumn{2}{c|}{ Dual-energy } & \multicolumn{2}{c}{ Monochromatic } \\
\hline & $Z_{\text {eff }}$ & $\mathrm{RE}$ & $Z_{\text {eff }}$ & $\mathrm{RE}$ & $Z_{\text {eff }}$ & $\mathrm{RE}$ \\
\hline Aluminum & $12.9 \pm 0.01$ & 0.8 & $9.96 \pm 0.03$ & 23 & & \\
Acrylic & $6.70 \pm 0.54$ & 3.6 & $6.43 \pm 0.21$ & 0.6 & & \\
Water & & & & & 7.39 & 2.0 \\
\hline
\end{tabular}




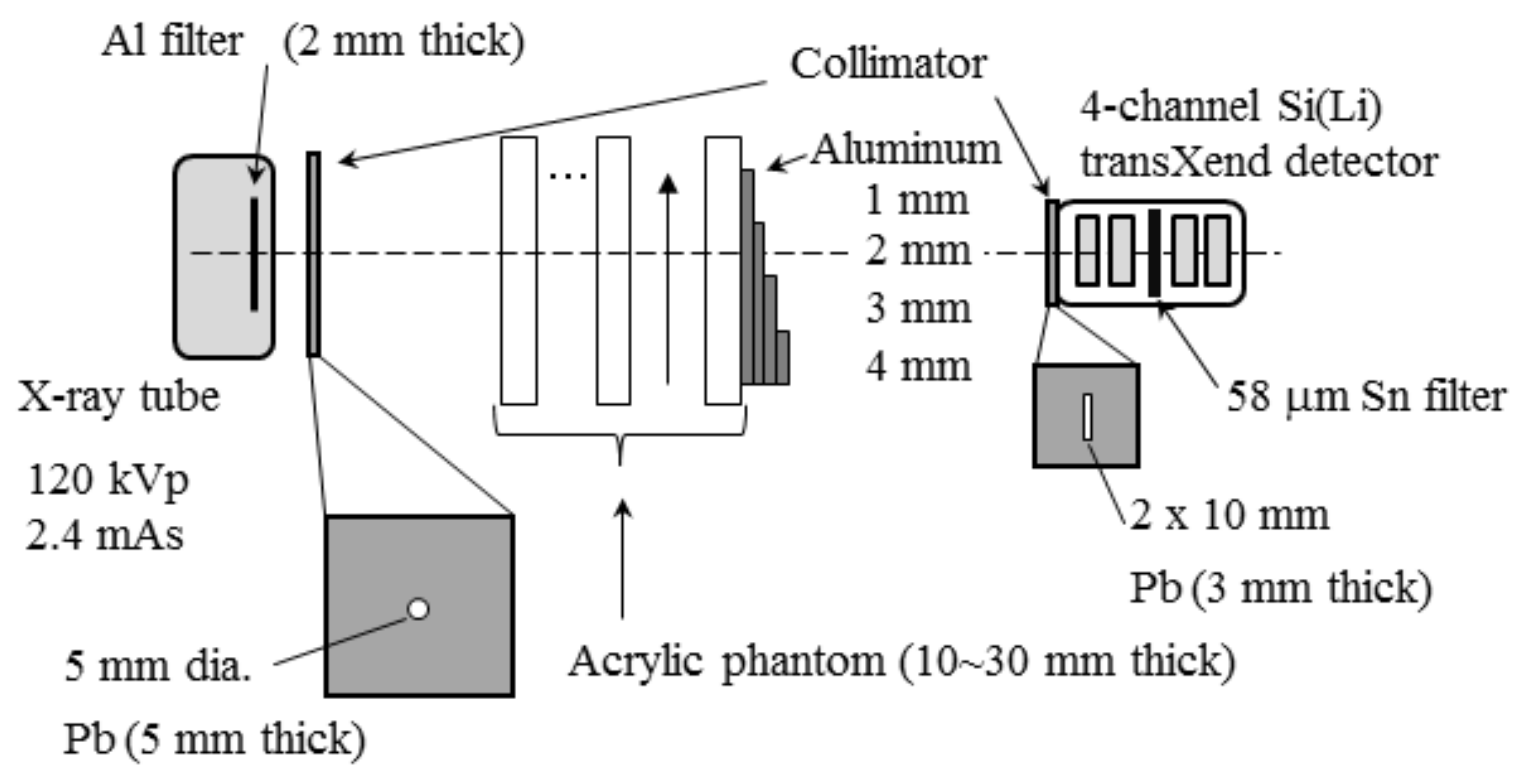

Figure 1. Experimental setup for response function estimation.

Y. Yamashita:

Measurement of effective atomic numbers using energy-resolved computed tomography 


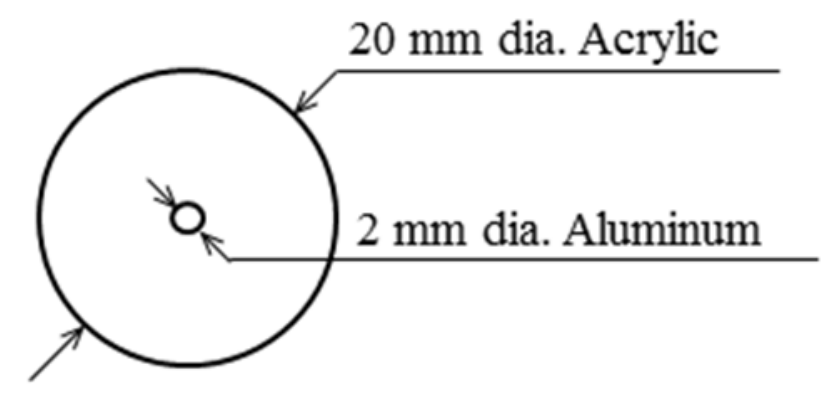

Figure 2. Schematic drawing of a cylindrical phantom.

Y. Yamashita:

Measurement of effective atomic numbers using energy-resolved computed tomography 


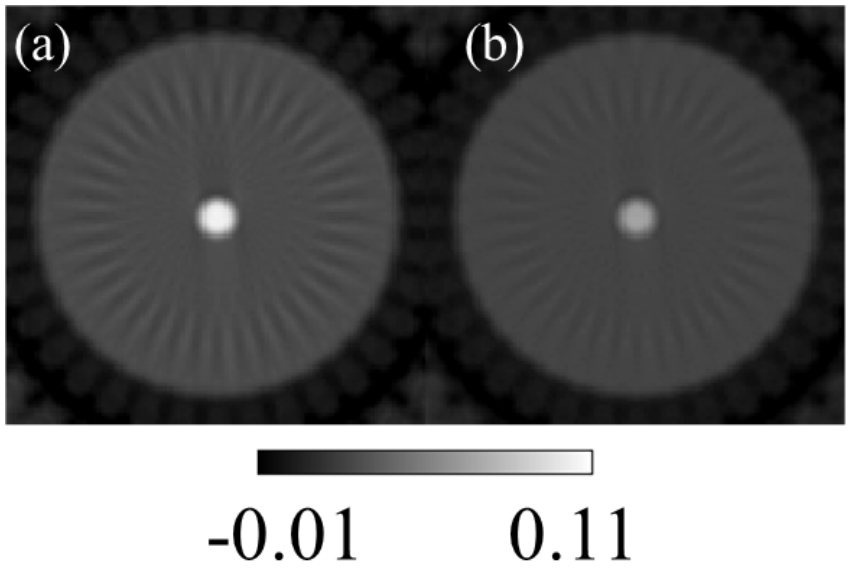

Figure 3. CT images obtained by X-rays in the energy ranges (a) $E_{2}$ and (b) $E_{5}$.

Y. Yamashita:

Measurement of effective atomic numbers using energy-resolved computed tomography 


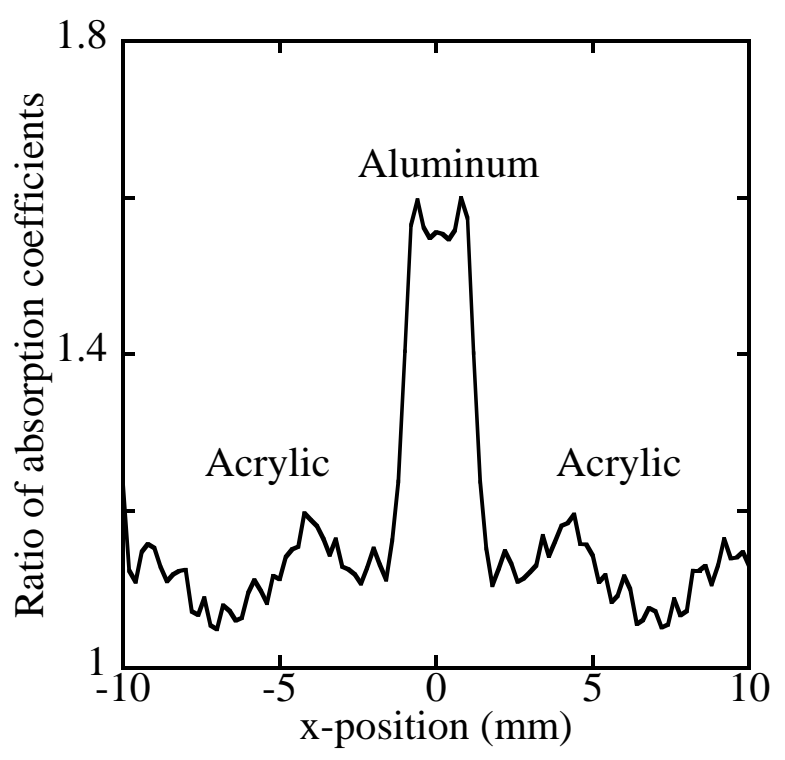

Figure 4. Profile of the linear attenuation coefficients ratio along the diameter of the phantom.

Y. Yamashita:

Measurement of effective atomic numbers using energy-resolved computed tomography 


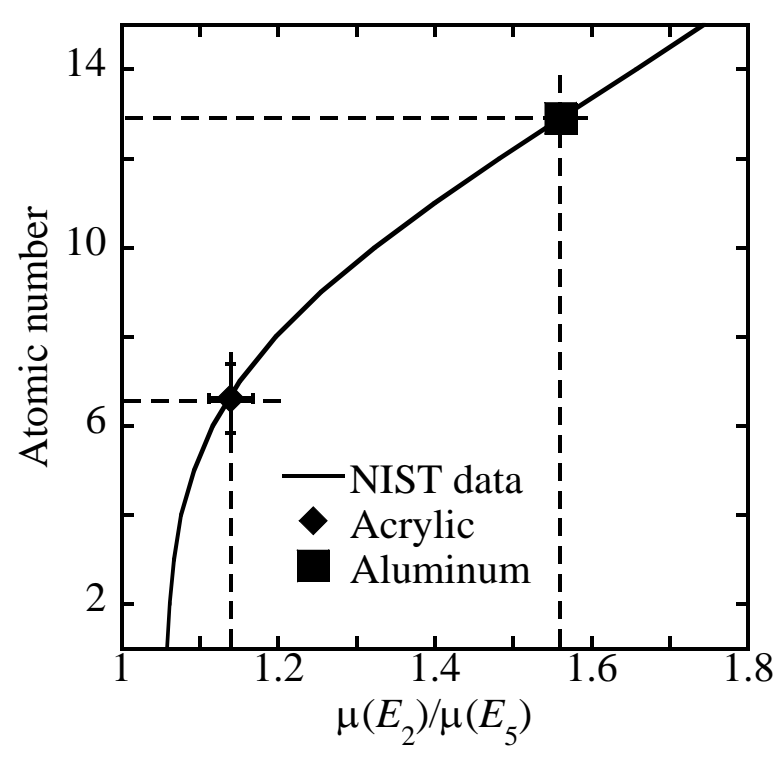

Figure 5. Relationship between $\mu\left(E_{2}\right) / \mu\left(E_{5}\right)$ and atomic number.

Y. Yamashita:

Measurement of effective atomic numbers using energy-resolved computed tomography 


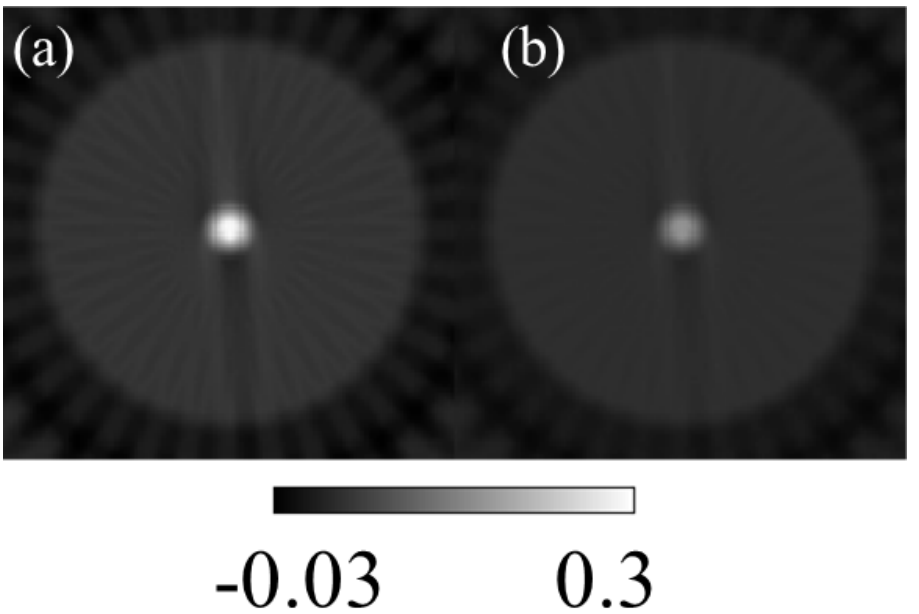

Figure 6. CT images obtained by current-measurement CT value with X-ray tube voltages of (a) $60 \mathrm{kVp}$ and (b) $120 \mathrm{kVp}$.

Y. Yamashita:

Measurement of effective atomic numbers using energy-resolved computed tomography 


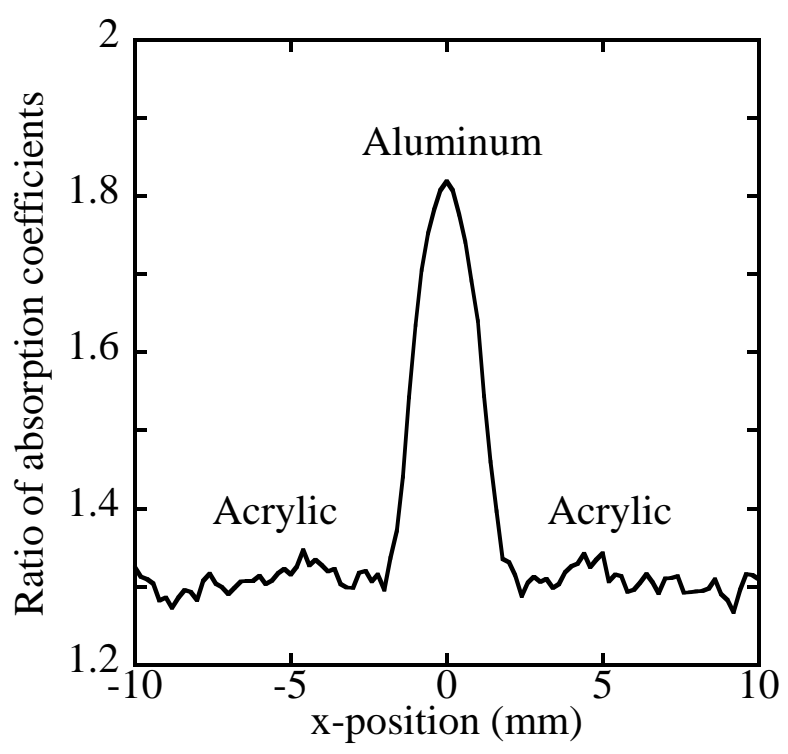

Figure 7. Profile of the linear attenuation coefficients ratio along the diameter of the phantom using dual-energy CT.

Y. Yamashita:

Measurement of effective atomic numbers using energy-resolved computed tomography 


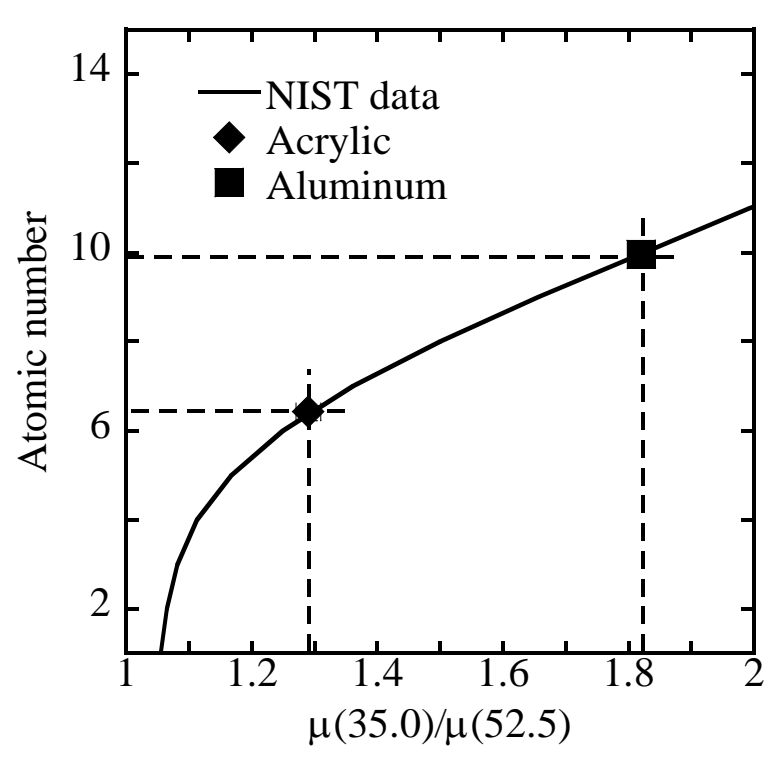

Figure 8. Relationship between the ratio of linear attenuation coefficients and atomic number using dual-energy CT.

Y. Yamashita:

Measurement of effective atomic numbers using energy-resolved computed tomography 


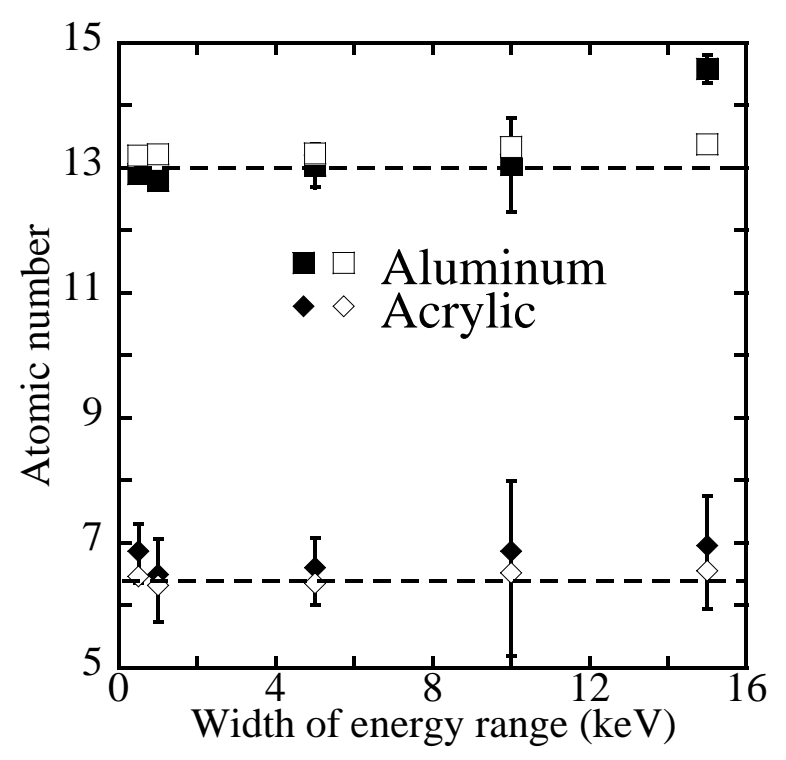

Figure 9. The effective atomic numbers as a function of those obtained by experiments (solid) and simulation calculation (open) energy range width for aluminum (squares) and acrylic (diamonds).

Y. Yamashita:

Measurement of effective atomic numbers using energy-resolved computed tomography 